\title{
LA COMPETITIVIDAD MACROECONÓMICA EN LA CONSTRUCCIÓN DE UNA REGIÓN DEL CONOCIMIENTO EN MÉXICO
}

\author{
Gina Laura María de Lourdes Baena Paz*, Salvador Neme Calacich**
}

\author{
Baena-Paz G.L.M.L., Neme-Calacich S. La \\ Competitividad macroeconómica en la construcción \\ de una región del Conocimiento en México. \\ Hitos de Ciencias Económico Administrativas \\ 2014;20 (56): 21-32.
}

\section{RESUMEN}

Objetivo: Conocer e identificar qué son las Ciudades del Conocimiento.

Material y métodos: Investigación cualitativa, exploratoria documental y entrevistas sobre las Ciudades del Conocimiento en México.

Resultados: Identificación de las Ciudades del Conocimiento en México.

Conclusiones: En México, aún es incipiente la noción sobre ciudades del conocimiento, entendiéndose muchas veces como clústers industriales, pero no se ha llegado a comprender la profundidad del concepto.

\author{
Baena-Paz G.L.M.L., Neme-Calacich S. The \\ macroeconomic competitiveness in the construction of \\ a knowledge region in Mexico. \\ Hitos de Ciencias Económico Administrativas
} 2014;20 (56): 21-32.

\section{ABSTRACT}

Objective: To determine and identify what are the Cities of Knowledge.

Material and methods: Qualitative research, exploratory documentary and interviews on Knowledge Cities in Mexico.

Results: Identification of Knowledge Cities in Mexico.

Conclusions: In Mexico, is still incipient notion of knowledge cities, often understood as industrial clusters, but has not come to understand the depth of the concept.
Palabras clave: Competitividad. Macroeconomía. Regiones del Conocimiento.
Key words: Competitiveness. Macroeconomics. Regions of Knowledge.

DIRECCIÓN PARA RECIBIR CORRESPONDENCIA: Correo electrónico: glbaenapaz@gmail.com

E

I nuevo orden económico mundial, la apertura de los mercados y la globalización de los medios de comunicación, han hecho más evidente que no sean sólo los países los que compiten, sino las regiones, e incluso las ciudades entre sí. La competitividad regional representa la nueva economía y quienes comprendan el cambio y logren adecuarse oportunamente a los nuevos enfoques serán los más competitivos, de ahí la trascendencia de identificar los sectores competitivos en cada región, como base para el desarrollo. A diferencia del pasado, en que los proyectos regionales expresaban un marcado énfasis en la planificación como instrumento de las políticas de desarrollo, en la actualidad, con la apertura y la globalización, el énfasis lo tienen los mecanismos de asignación del mercado en las políticas de desarrollo.

*Doctora en Gestión Estratégia y Políticas de Desarrollo. Profesora-Investigadora. DivisiónAcadémica de Ciencias Económico Administrativas (DACEA). Universidad Juárez Autónoma de Tabasco (UJAT).

** Doctor en Gestión Estratégia y Políticas de Desarrollo. Profesor-Investigador. DACEA-UJAT.

Fecha de recibido: 21 de noviembre de 2013 Fecha de aceptación: 10 de enero de 2014. 
En este sentido, el presente artículo pretende identificar la manera de cómo lograr la construcción de una Región del Conocimiento en México, en base a la competitividad macroeconómica.

\section{Material y método}

Realizando en primera instancia una breve descripción del concepto de competitividad y algunas acepciones de la misma; como parte dos, se planteará la caracterización de las Regiones del Conocimiento dentro de la función de producción a través de la variable sector externo, enmarcada en la competitividad regional; en la parte tercera se mostrarán los índices característicos de competitividad y de construcción de una Región del Conocimiento por cada Estado de México y se concluirá con algunas recomendaciones.

\section{Competitividad}

La competitividad es un término que se habla en todos los niveles, tanto microeconómico como macroeconómicamente, la cual de acuerdo a (Porter, 1986), constituye un factor determinante en el desarrollo económico del país, así mismo se habla de competitividad regional y territorial, teniendo como base las regiones productivas.

A nivel micro, la competitividad se define en función de su habilidad para obtener utilidades que se manifiestan en reinversión; lo anterior, no significa solamente maximizar beneficios a través de la minimización de costos, sino la combinación de cinco elementos (Best, 1990):

1) Dirección y gestión de empresa.

2) Introducción de innovaciones en el proceso productivo, en la mercancía o en la organización interna.

3) Coordinación en las fases del proceso de producción.

4) Organización de la industria en cuanto a competencia y cooperación .

5) Aprovechamiento de una política industrial activa que coadyuve el uso creativo y formativo del mercado, el enfoque de producción opuesto al de distribución y la determinación de sectores estratégicos y prioritarios para maximizar el crecimiento industrial.
También se habla de competitividad sistémica (Esser, Hillebrand, Messner, \& Meyer-Starner, 1994) la cual constituye un marco de referencia para países tanto industrializados como en vías de desarrollo. La visión de mediano a largo plazo y la intensa interacción entre los actores, no debe encaminarse únicamente a optimizar potenciales de eficacia en los diferentes niveles del sistema, movilizando capacidades sociales de creatividad con el fin de desarrollar ventajas competitivas nacionales. Ello ocurre porque ningún país puede escoger políticas o elementos de competitividad a partir del juego de determinantes niveles del sistema y su conducción (Delgado, 2006).

A nivel internacional el World Competitiveness Yearbook (WCY, 2009) define a la competitividad como «la habilidad de una nación para crear y mantener un entorno que sustente una mayor creación de valor para sus empresas y más prosperidad para sus habitantes" y para el WEF Global Competitiveness Report (Schwab, 2011) es «la habilidad de un país para alcanzar altas y sostenidas tasas de crecimiento del producto Per cápita».

A nivel nacional, la escuela de graduados en Administración Pública del ITESM y el Instituto Mexicano para la Competitividad (IMCO), es definida como «la habilidad de una región para atraer y retener inversiones" (Unidad de Planeación e Inversión Estratégica, 2007).

Con referencia al ranking mundial, de acuerdo al Índice de Competitividad de crecimiento (ICC) que desarrollaron (Sachs \& Warner, 1997) este mide los factores que contribuyen a sostener en el mediano plazo una tasa elevada del crecimiento del PIB Per cápita, tomando en cuenta criterios tanto anivel de empresa (microeconómico) como a nivel agregado (macroeconómico).

En el caso de México ocurre lo que se denomina la «Paradoja de la Competitividad»(Villareal \& R de Villareal, 2003) ya que es uno de los países más abiertos y de los menos competitivos, mantiene 31 acuerdos de libre comercio con países de 3 continentes y su índice de apertura al exterior es de 70\% (importaciones más exportaciones respecto al PIB). Sin embargo, de acuerdo al Informe Mundial de competitividad del Foro 
Económico Mundial, la baja competitividad de México lo ubica en el lugar 43 entre 59 países.

Saschs y Warner, también crearon el tríangulo de competitividad del crecimiento basado en tres indicadores; el índice de apertura al exterior, el índice de competitividad del sistema financiero y el índice de creatividad económica (Villareal \& R de Villareal, 2003) (ver figura 1).

\section{La competitividad macroeconómica y las regiones del conocimiento}

El nuevo esquema económico ya no es protagonizado por el Estado, son las empresas agrupadas en ciudades y regiones las que constituyen el motor del nuevo sistema económico que prevalece. De acuerdo a (Guajardo, 2006) las ciudades cobran gran importancia por ser espacios que albergan características que facilitan el funcionamiento de las empresas en el ámbito global.

El desarrollo regional se basa en identificar los mecanismos a través de los cuales las regiones pueden crecer en función de las actividades económicas localizadas en ellas (Macìas, 2004). Los estudios de tipo regional se sitúan en la parte intermedia entre el nivel micro de las decisiones de las universidades productivas y el macro de la política nacional, donde la región presenta rasgos homogéneos en su interior, los cuales deben ser observados conjuntamente, pero a la vez heterogéneos con relación a otras zonas.

Según (Sobrino, 2005), el estudio de la competitividad regional requiere metodologías distintas respecto al de la competitividad de las empresas, no sólo por los distintos objetivos, sino además por el efecto del desempeño económico de un territorio en el bienestar de su población residente, las responsabilidades y funciones que asume el sector público en el fomento y promoción del crecimiento económico.

La búsqueda de la competitividad en las regiones urbanas, tiene su origen en los objetivos políticos locales y nacionales. La intervención del gobierno en las ciudades, está progresivamente combinando acciones remediales con el propósito de combatir las consecuencias negativas de la urbanización y realice acciones proactivas para reforzar la competitividad. De acuerdo a (Kamal-Chaoui \& Spiezia, 2004) se presentan las siguientes políticas tendientes a intensificar la competitividad:

1) Apoyo a las Asociaciones para elevar el capital social local.

2) Vinculación entre instituciones de educación superior, instituciones de investigación,

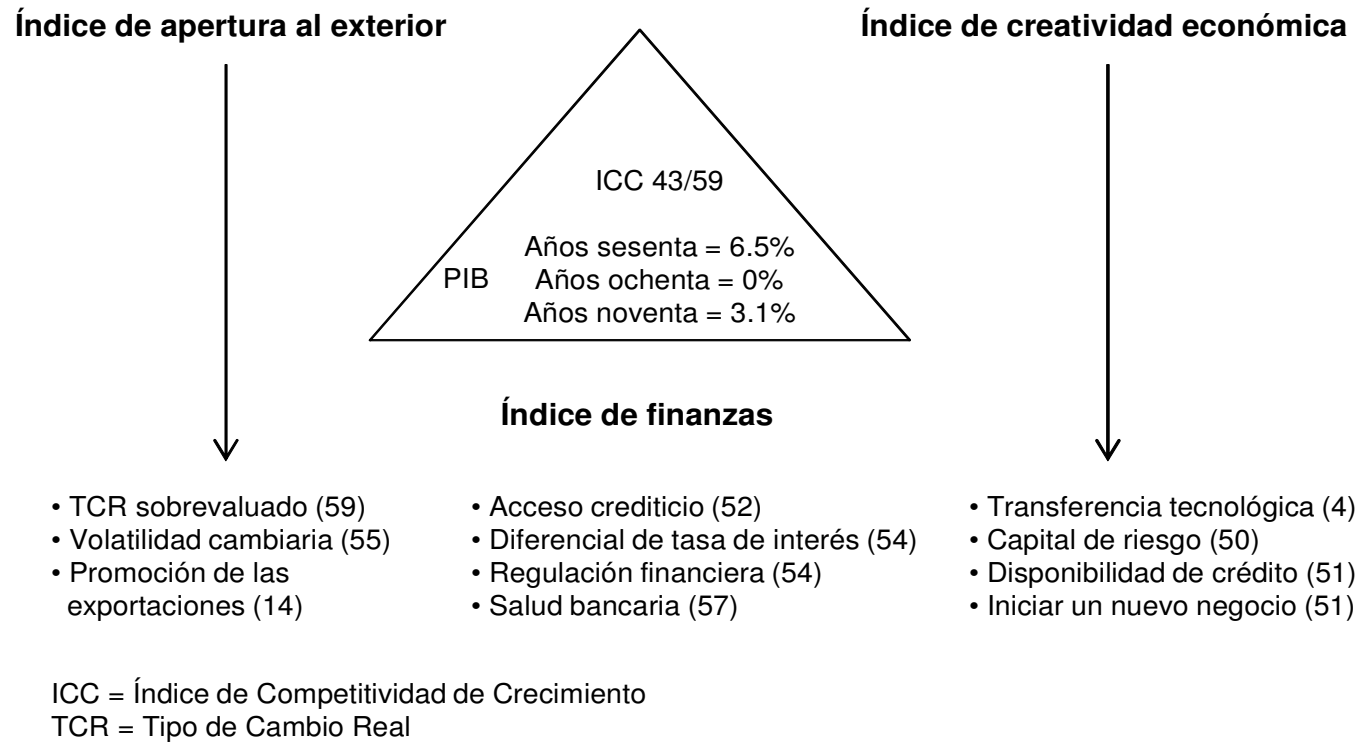

Figura 1.Triángulo de la Competitividad. Villareal \& R de Villareal, 2003 
industrias privada, gobierno y la provisión de capital humano cualificado que pueda operar efectivamente en el mundo del conocimiento.

3) Reforzar la comunicación (carreteras, aeropuertos y comunicaciones electrónicas).

Con respecto al desarrollo regional, debe abordarse bajo el enfoque de las capacidades competitivas de los sectores pivote o soporte de la actividad económica del lugar. Una vez identificados, se pone atención al efecto multiplicador que éstos puedan generar sobre el resto de las actividades económicas. El nuevo rol del Estado debe ser el de agente promotor y facilitador del desarrollo de la competitividad, en la cual los actores y gestores de la misma son los sectores involucrados; el sector público, el privado y el académico, a través de sus instituciones y sus líderes regionales. Esto es lo que constituye un proyecto de competitividad regional y su realización requiere el esfuerzo conjunto y la coordinación de todos (Indacochea, 1998) (ver figura 2).
Mientras más sólidas sean las estrategias regionales de inserción en el mundo globalizado, más sólido erá el avance hacia la competitividad del país. De acuerdo con este concepto, es imprescindible que los diferentes actores regionales comprendan la trascendencia e importancia del reto de la competitividad, para lograr una visión compartida para la región e identificar los sectores actuales y potenciales en que pueden ser competitivos, definiendo así la dirección estratégica en la cual es necesario avanzar para lograr la competitividad regional.

El aspecto macroeconómico es uno de los elementos que determinan el nivel de competitividad de una economía, seguido del aspecto microeconómico, la calidad de las instituciones y la capacidad para introducir cambios tecnológicos. Al brindar certidumbre a los distintos actores económicos (trabajadores, sindicatos, empresas), se constituye como la base sobre la cual se complementan los demás elementos.

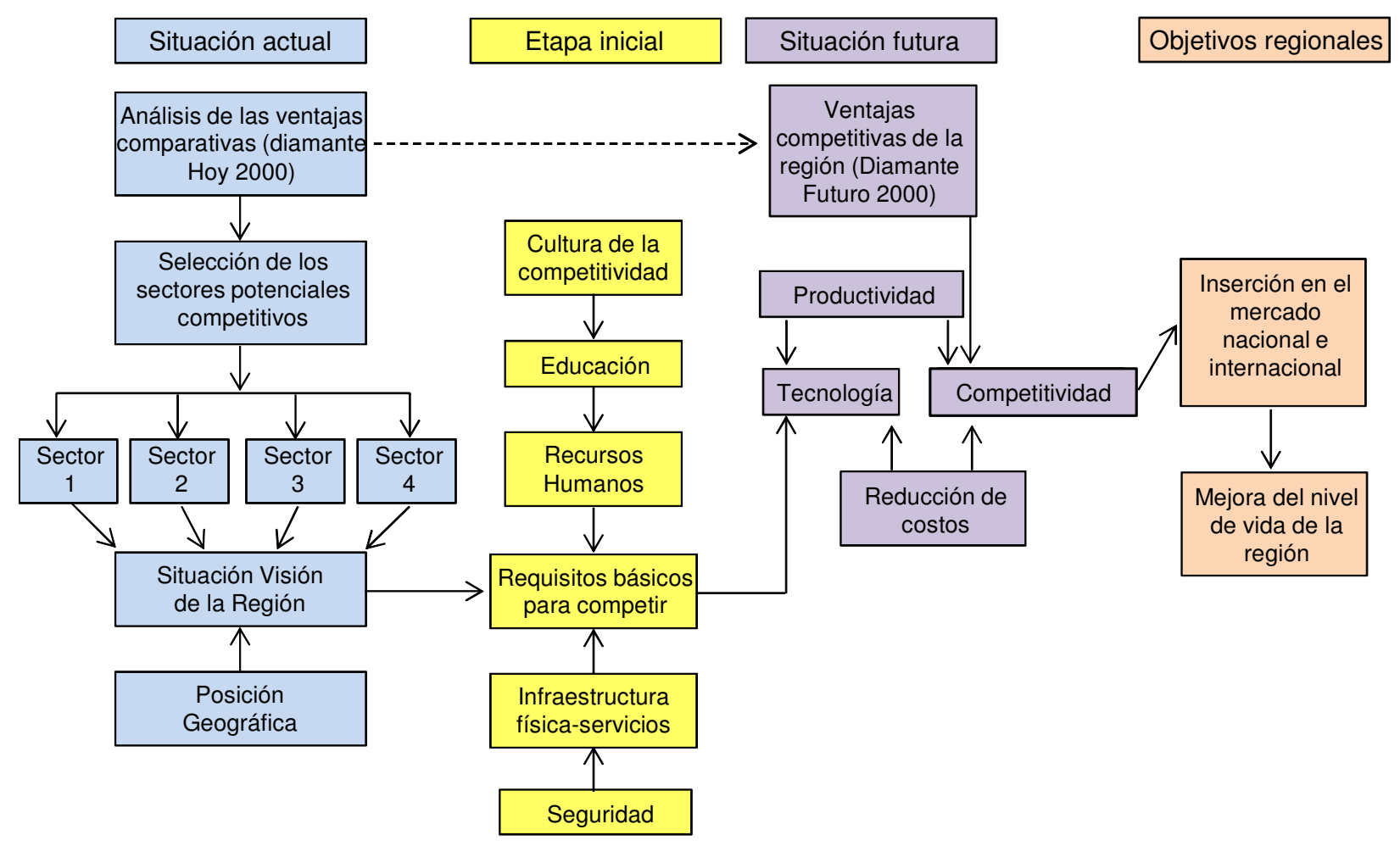

Figura 2. Proceso de Desarrollo de la Competitividad Regional. Alejandro Indacochea y colaboradores, «Cajamarca Competitiva». Lima, Mayo 1998, p. 203 
Si bien es cierto que la macroeconomía no crea riqueza, a diferencia de las empresas en el terreno microeconómico, sí puede destruir la riqueza y las empresas, de ahí que un inadecuado manejo macroeconómico con ausencia de una competitividad sistémica puede dar origen a errores, como por ejemplo de política cambiaria con peso fuerte y un dólar subsidiado que sobrevaluan o aprecian el tipo de cambio real. Los siguientes indicadores dan cuenta del comportamiento del entorno macroeconómico nacional y estatal y su repercusión cualitativa en la competitividad.

El nuevo concepto de la ventaja competitiva hace énfasis en los espacios regionales como los nuevos protagonistas del desarrollo, lo cual motiva su estudio por medio de abordajes como sistemas regionales de innovación, redes de innovación, redes de conocimiento, hélice triple o triple hélix (Leydesdorff \& Etzkowitz, 1998), entre otros, (ver figura 3).

Se ha re-configurado un nuevo modelo productivo, pasándose de un modelo basado en la producción y economías de escala, a otro que se basa en la distribución y economías de amplitud según los requerimientos de un mercado con exigencias crecientes.
La nueva ventaja competitiva de una región es el conocimiento disponible en la misma, las formas en las que se facilite el intercambio de conocimiento, capacitación y aprendizaje regional, cadenas productivas (en algunos sectores), financiamiento disponible, infraestructura y telecomunicaciones. Se espera también que exista una visión de futuro compartida por los actores, para que las estrategias de desarrollo sean sostenibles en el tiempo.

Esta tendencia, se ha visto reflejada en la formación de diversos conglomerados de empresas - espontáneas o inducidas - que fomentan las economías de aglomeración y la intensidad de las interacciones (Corona , 2005). Dentro de estas interacciones, se hará énfasis en aquellas que procuran y/o facilitan el flujo del conocimiento, ya que el conocimiento, conjuntamente con la creatividad, es el mayor insumo para la innovación y el desarrollo de nuevos productos.

\section{Se crean formas de aglomeración regional \\ - Polo tecnológico}

Un polo tecnológico consiste en un conglomerado de empresas especializadas ubicadas en una determinada región.

- Parque tecnológico

Un parque tecnológico se constituye en un conglomerado de empresas de diferentes sectores planeado con anterioridad y con el desarrollo de un proyecto arquitectónico, una orientación y caracterización del parque previo.

\section{- Clúster}

Los clúster son aglomeraciones formadas de manera espontánea por empresas de un determinado sector económico. En relación a los clúster(Porter, 1986) sostiene, coincidiendo con (Altenbur \& Meyer, 1998) son comunes entre empresas pequeñas tradicionales y en actividades intensivas en mano de obra. Se trata de una fábrica social basada en los valores compartidos mediante interacciones, donde se facilita la difusión del conocimiento e innovación. Ha sido identificada la escasez de espíritu emprendedor, falta de confianza y

Figura 3. Modelo Triple Hélice. Universia, 2005 
barreras de la información compartida, como los mayores cuellos de botella de un clúster.

De ellas se parte el concepto de Polo de Innovación Tecnológica, que consistiría en los aglomerados que dieran fuerza y rumbo a la creación y aplicación de conocimiento encaminado al desarrollo económico de la región, o lo que es lo mismo a la construcción de una región del conocimiento.

Dentro de los aspectos identificados en los Polos de Innovación es:

\section{a) Innovación}

La innovación está cada vez mas vista como un proceso sistémico en el cual participan múltiples agentes. Así la empresa para buscar el conocimiento necesario para el desarrollo de nuevos productos recurre a interacciones con otras empresas, proveedores, universidades, instituciones de investigación, entre otras.

(Peters, 2006)resalta la importancia que tiene para la sobre vivencia de las empresas, alejarse de «Commodities", por medio de adición de valor mediante los procesos de innovación continua.

El conocimiento obtenido por las empresas a través de sus interacciones con organizaciones de su entorno se constituye en el elemento crucial para la innovación de servicios, procesos y productos.

También es importante resaltar que son considerados artefactos característicos de la sociedad del conocimiento el computador y la microelectrónica, elementos que se encuentran o tienen aplicación en cualquier industria o servicio diluyendo de esta forma la diferenciación entre «high tech y low tech». (Fujii \& Huffman, 2006).

\section{b) Conocimiento tácito y conocimento articulado}

El conocimiento tácito es el que se ha acumulado durante un tiempo y es resultado de las practicas llevadas a cabo en una empresa o en una organización de I\&D, solo se puede transferir por medio de la interacción personal entre el «maestro y el aprendiz». Tal conocimiento se da por medio de lecciones: es práctico y es adquirido en el que hacer diario (aprender haciendo).
El conocimiento articulado o explicito es el disponible en manuales, en los documentos de las organizaciones, en los textos. Es susceptible de adquisición por medio de la lectura y análisis de documentos. Es importante, señalar que es la naturaleza del conocimiento lo importante y no la forma como es utilizado o archivado. (Gonzalez, 2005).

\section{Región del Conocimiento en México}

En un contexto global cambiante donde la globalización ha significado el resurgir de las regiones como elementos fundamentales en la generación del conocimiento que caracteriza a la nueva economía, se hace cada vez más necesario el contar con herramientas para analizar esta problemática.

El grupo de los países más industrializados G7 consideró que el beneficiario último de la sociedad de la información y el conocimiento, debe ser el ciudadano, por lo tanto, las políticas que se diseñen tienen que conservar como objetivos fundamentales mejorar la calidad de vida y crear empleos(Warkentin de la Mora, 2003).

Por ello, se puede decir que una Región del Conocimiento es un propósito social: que su ciudadanía tenga buena calidad de vida, bajo nivel de desempleo, un considerable nivel de cohesión social, y consolidados proyectos de transformación e innovación que impulsen nuevas perspectivas de desarrollo económico regional.

\section{INDICADORES BASES PARA LA CONSTRUCCIÓN DE} UNA REGIÓN DEL CONOCIMIENTO

Se definen cinco variables básicas que influyen en la construcción de una Región del Conocimiento(ITESM, 2003):

\section{1.-Capital humano}

Este se entiende como la capacidad de aprendizaje y conocimiento adquirido de una población, este se puede medir a través del porcentaje de población con alguna instrucción educativa, así para construir este indicador se utilizó:

- Población de 15 años y mas que sabe leer y escribir.

- Población con instrucción pos primaria.

- Población con instrucción media superior y superior. 
- Población con instrucción superior.

- Población con maestría o doctorado.

Con lo anterior, se calculó el índice de capital humano por Entidad Federativa.

\section{2.- Investigación e innovación}

Se entiende por investigación e innovación el grado de investigación, tecnologías e innovaciones en cada Entidad Federativa y se determinó en base a:

- Integrantes del SIN.

- Centros de Investigación.

- Solicitudes de Patentes.

- Becas CONACYT.

\section{3.- Empresarialidad}

Este concepto se basa en el desarrollo empresarial que se presenta en cada entidad federativa y se determinó considerando:

- El número de empresas registradas.

- El número de empresas certificadas.

Con esto se calculó el índice de empresarialidad por entidad federativa.

\section{4.- Gestión y comunicación}

Aquí se considera el nivel de comunicación entre los hogares, las empresas y las personas, así como la gestión de trámites, con ello se consideró:

- Líneas telefónicas por cada mil habitantes.

- Índice de calidad en la gestión de trámites empresariales.

- Porcentaje de hogares con internet.

Así se obtiene el índice de comunicación y gestión.

\section{5.- Tecnologías de Información y Comunicación}

Es el grado tecnológico para el ambiente de obtención de información y su transformación en conocimiento, así se considera:

- La matrícula de estudiantes en áreas de computación y sistemas.

- Los activos fijos en TIC.

- El número de empresas en la industria de la TIC.

- Número de escuelas de nivel superior que imparten computación y sistemas.

Con ello, se construyó el índice de TIC.

En la tabla 1, se presenta los índicadores de Crecimiento Nacional por Estado(INEGI, 2001).
En la tabla 2, se presentan los indicadores de la Región del Conocimiento, elaborada por (ITESM, 2003).

\section{Resultados}

En base a estos estudios, se tienen proyectado actualmente 10 ciudades del conocimiento en México, como son:

1. Monterrey, se ha trabajado de manera importante en el Parque de Innovación e Investigación Tecnológica, con una inversión de 1,000 millones de pesos para un espacio de 70 hectáreas, donde operan actualmente 10 centros de investigación -y otros 10 en construcción que serán finalizados esté año- con más de 650 investigadores en 10 clúster específicos: producción automotriz, enseres domésticos, tecnologías de información y software, servicios médicos y ciencias de la vida, biotecnología, nanotecnología, agroindustria, vivienda y desarrollo sustentable, aeronáutica y multimedia (Corona Treviño, Innovación y Región, 2001) (Carrillo, 2008) (Ramírez, 2011).

2. Biometrópolis, Distrito Federal, ubicado en un predio de 71 hectáreas, articulará y potenciará una masa crítica de investigadores de alto nivel, que permitirá un avance cuántico en el desarrollo tecnológico a nivel mundial, creando nuevos empleos para los médicos y científicos mexicanos. (UNAM, 2001) (Skyscraper, 2009) (UNAM, 2009) (Ramírez, 2011).

3. Guadalajara, el «Valle del silicio» mexicano, donde compañías de primer nivel en materia de electrónica como Intel, Siemens o Flextronics han incrementado y ampliado operaciones, es un referente de aprovechamiento de la industria generada a partir de la innovación en el saber humano (Ramírez, 2011).

4. Mérida, la Fundación Plan Estratégico de Yucatán, coordinó en conjunto con el Centro de Investigación Científica de Yucatán, la ejecución del proyecto «Mérida Ciudad Internacional del Conocimiento", para lo cual contó con la invaluable participación de especialistas en diversas disciplinas de las más importantes instituciones de educación superior en la entidad, así como representantes de empresas, de los gobiernos estatal, municipal y federal, cámaras empresariales, colegios de profesionales y centros de investigación. (Gobierno de Yucatán, 2010) (Ramírez, 2011). 


\begin{tabular}{|c|c|c|c|c|c|c|c|c|c|}
\hline ENTLAD & $\begin{array}{l}\text { Crecimeitno } \\
\text { económio }\end{array}$ & 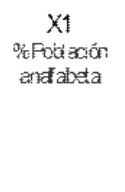 & $\begin{array}{c}\times 2 \\
\text { \%potiacion } \\
\text { sinprimaria } \\
\text { competa }\end{array}$ & $\begin{array}{c}\text { X3 } \\
\text { o Oupates } \\
\text { Giviendas } \\
\text { sinderbie ni } \\
\text { sevido } \\
\text { sataro }\end{array}$ & $\begin{array}{c}\times 4 \\
\text { cociparie } \\
\text { envivigdes } \\
\text { sin enagia } \\
\text { jedrica }\end{array}$ & 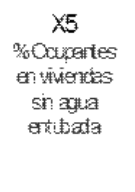 & $\begin{array}{l}\text { \%oviendas } \\
\text { con aigin } \\
\text { rifd de } \\
\text { recromiero }\end{array}$ & $\begin{array}{l}\text { vodipertes } \\
\text { en vivendas } \\
\text { enposo de } \\
\text { tiera }\end{array}$ & 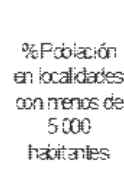 \\
\hline Nacional & $278 \%$ & 837 & 23,10 & 5.34 & 2,49 & 10,14 & 40,64 & 11,48 & 289 \\
\hline Aguascalientes & $4,50 \%$ & 4,16 & 17,82 & 1,58 & 0,85 & 1,79 & 34,34 & 2,36 & 24,67 \\
\hline Eaja Calfonia & $395 \%$ & 308 & 15,02 & 0.56 & 1,49 & 4,89 & 32,3 & 3,95 & 9.27 \\
\hline Baja Califomia Su & $4.78 \%$ & 362 & 16,49 & 1,84 & 288 & 11,28 & 35,16 & 8,27 & 19.84 \\
\hline Campeche & $1,92 \%$ & 1020 & 26,96 & 9.85 & 486 & 11,15 & 51,42 & 8,77 & 32,01 \\
\hline Ccahulla de Zaragcza & $4,30 \%$ & 3,29 & 14,60 & 1,65 & 0.77 & 2,17 & 33,71 & 2,51 & 12,33 \\
\hline colima & $3,13 \%$ & 642 & 21,58 & 0.80 & 0,67 & 1,66 & 34,92 & 8,40 & 15,78 \\
\hline Giapas & $2,32 \%$ & 21,35 & 42,76 & 8.07 & 588 & 25,90 & 60.20 & $32 \%$ & 58,46 \\
\hline Crintahua & $3,97 \%$ & 4,42 & 18,81 & 3,29 & 4,28 & 6,45 & 31,93 & 5.45 & 17.54 \\
\hline Distrito Fecieral & $1,22 \%$ & 259 & 9,70 & 0.16 & 0,15 & 1,51 & 29.31 & 1,12 & 0.52 \\
\hline Durango & $3,92 \%$ & 484 & 22,92 & 8,51 & 3,52 & 8,63 & 35,09 & 11,34 & 38,64 \\
\hline Guanajuato & $3.60 \%$ & 1044 & 28,80 & 9.87 & 1,98 & 6,14 & 40,90 & $8 \% 5$ & 34,46 \\
\hline Querero & $1,40 \%$ & 1988 & 35,98 & 27,18 & 6,33 & 31,34 & 55,06 & 35,69 & 50.51 \\
\hline Hcialgo & $1,95 \%$ & 1280 & 27,50 & 8.98 & 3,90 & 12,21 & $42, \oplus$ & 12,78 & 57,25 \\
\hline Jalisco & $2,13 \%$ & 556 & 21,30 & 2,42 & 1,12 & 5,91 & 33,27 & 5.35 & 17.40 \\
\hline Mexico & $2,80 \%$ & 5.32 & 16,24 & 4,76 & 0,96 & 6,04 & 41,46 & 6,04 & $18, \infty$ \\
\hline Mchoacán de Ccampo & $2,01 \%$ & 1258 & 33,48 & 6,66 & 2,11 & 9,97 & 40,01 & 15,67 & 40,61 \\
\hline Mbrebs & $297 \%$ & 813 & 21,01 & 3,10 & 0.81 & 7,84 & $38, \infty$ & 10,71 & 228 \\
\hline Nayarit & $2,21 \%$ & 802 & 26,05 & 6,78 & 4,38 & 8,35 & 37,73 & 9.93 & 41,67 \\
\hline Nevo Lech & $4.35 \%$ & 278 & 12,70 & 0,54 & 0,56 & 3,48 & 33,40 & 2,34 & 7,13 \\
\hline Caxaca & $1.69 \%$ & 19.35 & 38,49 & 6,84 & 7.21 & 26,29 & 53,05 & 35.17 & 51.77 \\
\hline Pueda & $285 \%$ & 1271 & 29,02 & 5,45 & 2,19 & 14,03 & 49,16 & 15,86 & $3, \infty$ \\
\hline Querétao & $3,89 \%$ & 814 & 20,03 & 9,95 & 2,99 & 5,76 & 37,60 & 8,59 & 38,34 \\
\hline Ointana Roo & $4,11 \%$ & 6,58 & 19.42 & 5,19 & 259 & 4,66 & 49,90 & 8,41 & 17,10 \\
\hline San Luis Potosi & $4,65 \%$ & 992 & 27,42 & 5,72 & 5,58 & 16,97 & 38,20 & 19,67 & 41,18 \\
\hline Snaba & $2,27 \%$ & 642 & 23.42 & 5,14 & 1,92 & 6,24 & 43,08 & 9,78 & 35,30 \\
\hline Sonora & $3,70 \%$ & 3.73 & 17,21 & 1,92 & 1,87 & 4,01 & 38,73 & 9,67 & 18,2 \\
\hline Tabasco & $1.92 \%$ & 867 & 25,10 & 3,99 & 1,95 & 2294 & 47,11 & 9.11 & 56,78 \\
\hline Tamatipas & $4,16 \%$ & 4,52 & 18,61 & 0,84 & 288 & 4,26 & 39,24 & 5,39 & 149 \\
\hline $\begin{array}{l}\text { Tlaxcala } \\
\text { Veracnz de lgnacio dela }\end{array}$ & $2,02 \%$ & 668 & 18,78 & 4,84 & 1,11 & 2,03 & 47,93 & 5,26 & 40,30 \\
\hline Lave & $291 \%$ & 1342 & 32,90 & 4.18 & 4,67 & 23,32 & 45.02 & 2,77 & 46.68 \\
\hline Yucatán & $3,31 \%$ & 1089 & 29.99 & 17,96 & 261 & 3.03 & 48,35 & 4,81 & 27,42 \\
\hline Zactecas & $3.60 \%$ & 7.20 & 30.83 & 10.53 & 1.91 & 6,72 & 37,06 & 6.29 & 50.30 \\
\hline
\end{tabular}

Nota: Elaborado con base en INEGI, 2001.

5. Guanajuato, la Expo Guanajuato Bicentenario, en la que se gastaron mil 100 millones de pesos para festejar la Independencia, se transformará en un Parque Tecnológico, que el gobierno estatal presume como «la Ciudad del Conocimiento». Integrará a la Universidad de Guanajuato, Consejo Nacional de Ciencia y Tecnología, la UNAM, el IPN y a los tecnológicos de León y Celaya (Corona, 2001) (UPN, 2010) (Ramírez, 2011).

6. Ciudad del Conocimiento Nayarita C2N, la ciudad del conocimiento nayarita es una de las estrategias del gobierno del estado y del CONACYT para el fortalecimiento de las capacidades científicas y tecnológicas locales y regionales. (Gobierno de Nayarit, 2010) (Ramírez, 2011).

7. Santiago de Querétaro, «México está dentro de un ambiente de desarrollo aeroespacial y hay una madurez en la industria; la industria, es lo suficientemente madura para poder dar soporte al sector aeroespacial en el futuro» (Corona Treviño, Innovación y Región, 2001) (El corregidor, 2010) (Skyscrapercity, 2010) (Ramírez, 2011).

8. Torreón, el Museo Nacional de la Aviación y el Espacio, la Ciencia y la Tecnología, A.C., desde hace 11 años ha venido trabajando para hacer realidad la «Ciudad del conocimiento»; que consta de una 
Tabla 2

Indicadores para la región del conocimiento

\begin{tabular}{|c|c|c|c|c|c|}
\hline Estado & $\begin{array}{c}\text { Índice de } \\
\text { Investigación e } \\
\text { Innovación }\end{array}$ & $\begin{array}{c}\text { Índice de } \\
\text { Empresarialidad }\end{array}$ & $\begin{array}{c}\text { Índice de } \\
\text { Gestión y } \\
\text { Comunicación }\end{array}$ & $\begin{array}{c}\text { Índice de } \\
\text { TIC }\end{array}$ & $\begin{array}{c}\text { Índice de } \\
\text { Capital } \\
\text { Humano }\end{array}$ \\
\hline Aguascalientes & 4.24 & 2.6 & 7.41 & 3.1 & 7.16 \\
\hline Baja California & 5.12 & 6.6 & 6.04 & 7.1 & 8.77 \\
\hline Baja California Sur & 3.03 & 0 & 6.42 & 0.6 & 8.00 \\
\hline Campeche & 0.39 & 0.6 & 2.8 & 2 & 3.81 \\
\hline Chiapas & 3.23 & 4.3 & 1.48 & 4 & 0.00 \\
\hline Chihuahua & 2.88 & 6.1 & 6.19 & 7.4 & 6.39 \\
\hline Coahuila & 3.88 & 6.4 & 5.6 & 7.4 & 8.97 \\
\hline Colima & 3.07 & 0.7 & 5.08 & 1 & 6.90 \\
\hline Distrito Federal & 8.97 & 10 & 8.57 & 10 & 10.00 \\
\hline Durango & 3.74 & 1.5 & 5.89 & 2.4 & 4.77 \\
\hline Guanajuato & 6.92 & 7.9 & 4.77 & 6.1 & 2.58 \\
\hline Guerrero & 2.31 & 4.6 & 4.95 & 3.6 & 1.61 \\
\hline Hidalgo & 1.88 & 3.9 & 1.23 & 4.1 & 1.35 \\
\hline Jalisco & 6.01 & 8.9 & 4.42 & 8.5 & 5.23 \\
\hline México & 8.75 & 9.6 & 4.53 & 8.69 & 6.39 \\
\hline Michoacán & 6.07 & 6.3 & 4.66 & 4.2 & 1.48 \\
\hline Morelos & 6.83 & 2.6 & 6.98 & 3.9 & 6.65 \\
\hline Nayarit & 1.76 & 1.6 & 3.9 & 1.5 & 5.10 \\
\hline Nuevo León & 5.93 & 8.4 & 7.80 & 7.6 & 9.68 \\
\hline Oaxaca & 4.06 & 4 & 0.92 & 2.7 & 0.39 \\
\hline Puebla & 6.44 & 7.1 & 3.68 & 8.5 & 2.58 \\
\hline Querétaro & 6.19 & 3.9 & 7.36 & 3.7 & 5.61 \\
\hline Quintana Roo & 2.05 & 1 & 5.98 & 1.40 & 5.68 \\
\hline San Luis Potosí & 3.03 & 3.8 & 5.18 & 2.8 & 2.77 \\
\hline Sinaloa & 3.39 & 3.2 & 5.96 & 4.9 & 6.26 \\
\hline Sonora & 4.62 & 5 & 6.48 & 6.8 & 8.19 \\
\hline Tabasco & 1.26 & 2.2 & 2.46 & 4.6 & 3.29 \\
\hline Tamaulipas & 2.12 & 6.5 & 4.73 & 8 & 7.94 \\
\hline Tlaxcala & 2.36 & 1 & 5.98 & 2 & 4.19 \\
\hline Veracruz & 4.84 & 7 & 5.89 & 7.3 & 2.06 \\
\hline Yucatán & 5.11 & 2.8 & 4.62 & 3.6 & 3.74 \\
\hline Zacatecas & 1.52 & 2.6 & 2.76 & 0.8 & 2.45 \\
\hline
\end{tabular}

Nota: ITESM, 2003.

comunidad Científica, Tecnológica, Industrial y Comercial; de carácter y participación mexicana e internacional (Tu esfera, 2010) (Ramírez, 2011).

9. Cuernavaca, los 39 Centros de Investigación, sus 135 laboratorios y sus más de1,900 investigadores constatan el sueño del Estado de Morelos de convertirse en el Estado puntero en el ámbito tecnológico y científico (Corona, 2001) (El sol de Cuernavaca, 2008) (El mundo, 2008) (Ramírez, 2011).

10. Ciudad Juárez, en este caso se ha invertido 1,500 millones de pesos, el proyecto denominado Ciudad del Conocimiento opera en realidad como una ciudad universitaria, en la cual tienen instalaciones seis instituciones educativas, (Ramírez, 2011). 


\section{CONCLUSIONES}

El estudio de las Regiones del Conocimiento, aun es poco estudiado, pero su enlace con la Macroeconomía es de vital importancia por el incremento a la competitividad que esta se genera en el aumento de la productividad de cada Estado, para realizar exportaciones y así influir en la función de producción de la nación.
Es imprescindible utilizar las aportaciones de la nueva economía regional y generar una nueva forma de definir los rumbos de la nación, identificando claramente los sectores competitivos y diseñando sobre ellos el nuevo proyecto de país hacia una regionalización competitiva y especializada.

\section{REFERENCIAS}

Altenbur, H., \& Meyer, S. (1998). How to promote clusters? Polici experience from latin america. World Development, vol 27 n9.

ANFECA. (2001). Ciudad del Conocimiento. México: ANFECA.

Baena Paz, G. (2012). The University as a Strategic Manager in the Construction of a Region of Knowledge. Case Juarez Autonomus of Tabasco University Tabasc. IFKAD-WCKS 2012 (págs. 387-417). Matera, Italia: Institute of Knowledge Asset Management,Queensland University of Technology, University of Basilicata y World Capital Institute.

Baena Paz, G. (2013). La universidad como gestor estratégico en la construcción de una región del conocimiento: caso Universidad Juárez Autónoma de Tabasco. Tesis doctoral en Gestión Estratégica y Políticas de Desarrollo. Universidad Anahuac-Mayab. Mérida, Yucatán.

Baena Paz, G., \& Neme, S. (2013). The relationship university, business and government in the construction of a knowledge region in Tabasco. Mexico. Estambul, Turquia: KCWS.

Baena Paz, G., Neme, S., Martínez, L., \& Armenta, A. (2014). The «Region of Knowledge» initiative as planning model for economic growth in the SouthSoutheast region of Mexico . Papers IFKAD-2014 (pág. 20). Matera, Italia: IFKAD-2014.

Best, M. (1990). The new competition. Cambridge: Harvard University Press.
Carrillo, F. (2008). Ciudades de conocimiento: de la perspectiva internacional al caso Monterrey. Revista Colombiana de Telecomunicaciones, 4953.

Corona Treviño, L. (2001). Innovación y región. México: UNAM-IPN-CONACYT.

Corona Treviño, L. (2005). México: el reto de crear ambientes regionales de innovación. México: CIDE-FCE.

Delgado, F. A. (septiembre, 2006). Competitividad. Recuperado de la Universidad de El Salvador: www.monografias.com

El corregidor. (agosto, 2010). mcne. Recuperado de http://www.elcorregidor.com.mx/macne...otes/ ?id=68913

El mundo. (agosto, 2008). Cuernavaca ciudad del conocimiento. Recuperado de http:// www.elmundo.es/elmundo/2008/08/03/espana/ 1217726987.html

El sol de Cuernavaca. (julio, 2008). Cuernavaca, ciudad del conocimiento. Recuperado de http:// www.oem.com.mx/elsoldecuernavaca/notas/ n1994634.htm

Esser, K., Hillebrand, W., Messner, D., \& Meyer-Starner, J. (1994). Competitividad Sistémica. Berlin: Instituto Aleman de Desarrollo.

Fujii, D., \& Huffman, C. (mayo, 2006). El burro y la zanahoria: desempeño de los programas de estímulos fiscales en M'sxixo 2001-2005. Recuperado de http:// 
www.sociedadconocimiento.unam.mx/ elburroylazanahoria.pdf

Gobierno de Nayarit. (agosto, 2010). introducción. Recuperado de http:// www.cdconocimientonayarit.gob. $\mathrm{mx} /$ introduccion.php

Gobierno de Yucatán. (octubre, 2010). Plan estratégico de Mérida. Recuperado de http:// planestrategicodemerida.org. $m x / ? p=384$

Gonzalez, M. C. (mayo, 2005). Dicotomías para analizar el conocimiento tácito y el articulado. Recuperado de ghtc.ifi.unicamp.br/AFHIC3/.../38Maria-Cristina-Gonzalez.pdf

Guajardo, M. (2006). Reseña de ciudades del Siglo XXI ¿competitividad o cooperación? de Enrique Cabrero Mendoza Carlos Arce Macias y Alicia Ziccardi. Gestión y Política Pública, 201-206.

Indacochea, A. (mayo, 1998). El desafío de la competitividad regional. Recuperado de http:// palestra.pucp.edu.pe/index.php?id=80

INEGI. (2001). Tabulados básicos nacionales y por entidad federativa. Base de datos y tabulados de la muestra censal, XII censo general de población y vivienda. México: INEGI.

ITESM. (2003). Las regiones del conocimiento en México. Una metodología de análisis. Seminario de las regiones ante la sociedad del conocimiento del diagnóstico a las políticas (25-55). Tijuana: Seminario .

Kamal-Chaoui, L., \& Spiezia, V. (2004). Metropolitan governance and economic competitiveness. Review Revista de Economía Pública Urbana, 41-62.

Leydesdorff, L., \& Etzkowitz, H. (1998). La triple hélice como un modelo para estudios de innovación. Ciencias y Política Pública (25) 195-203. París: Ciencias y Política Pública.

Macìas, A. (2004). Crecimiento económico y competitividad en las regiones. Las ciudades medias de Jalisco, el caso de Zapotlán el Grande. Región y sociedad, 39-82.
Peters, T. (febrero, 2006). Tom Peters recomienda a las empresas españolas, la innovación constante como vía para mantenerse en el mercado. Recuperado de http://www.consultoras.org/ frontend/aec/Tom-Peters-Recomienda-A-LasEmpresas-Espanolas-La-Innovacion-ConstanteComo-Via-Para-Mantenerse-En-E-vn4289-vst199

Porter, M. E. (1986). Estrategia competitiva: técnicas para el análisis de los sectores industriales y de la competencia. Montevideo: CECSA.

Ramírez, E. (abril, 2011). Construyen 10 ciudades del conocimiento. Recuperado de http:// eleconomista.com.mx/sociedad/2011/04/11/ construyen-10-ciudades-conocimiento

Sachs, J., \& Warner, A. M. (noviembre, 1997). ciddata. Recuperado de www.cid.harvard.edu/ciddata/ warner_files/natresf5.pdf

Schwab, K. (2011). The global competitiveness report 2011-2012. Switzerland 2011: World Economic Forum.

Skyscraper. (diciembre, 2009). Proyecto ciudades del conocimiento campus biometropolis. Recuperado de http://www.skyscrapercity.com/ showthread.php?p=47194499

Skyscrapercity. (julio, 2010). showthread. Recuperado de http://www.skyscrapercity.com/ showthread.php?t=386303\&page $=5$

Sobrino, J. (2005). Competitividad territorial, ámbitos e indicadores de análisis. Economía, sociedad y territorio, 123-183.

Tu esfera. (octubre, 2010). aviación. Recuperado de http://tuesfera.com/aviacion/cdc/cdc.php

UNAM. (2001). Construccion de sociedades de conocimiento nuevos retos para la educacion superior. Perfiles Educativos, 1-17.

UNAM. (03 de 12 de 2009). Ciudad del conocimiento. Campus biometropolis. Recuperado de http:// www.biometropolis.mx/

Unidad de Planeación e Inversión Estratégica. (mayo, 2007). Competitividad y tipo de cambio. 
Recuperado de seip.guanajuato.gob.mx/.../427competitividad-y-tipo-de-cambio.

Universia. (junio, 2005). Un nuevo modelo: la triple hélix. Recuperado de http://investigacion.universia.es/ spin-off/triple-helice/index.htm

UPN. (abril, 2010). Guanajuato ciudad del conopcimiento. Recuperado de http:// anuario.upn.mx/index.php/noticias-educativas/ noticias-educativas-2010/72-el-universal/10227expo-guanajuato-se-convertira-en-ciudad-delconocimiento-.html

Villareal, R., \& R de Villareal, R. (septiembre, 2003). Agenda Cepal. Recuperado de www.cepal.org/ ddpe/agenda/7/8367/VillarrealRamos.pdf

Warkentin de la Mora, G. (2003). ¿Qué entendemos por sociedad de la información? El reto de México ante la Cumbre Mundial de la Sociedad de la Información. México: Fundación Konrad.

WCY. (septiembre, 2009). Anuario de competitividad. Recuperado de http://www.imd.org/research/ publications/wcy/index.cfm 